\title{
Research on Effective Integration of Kindergarten Curriculum and Game Jie Yin
}

\author{
Institute of Teacher Education, China West Normal University, Nanchong, 637000, China
}

\author{
Keywords: Kindergarten curriculum; game; effective integration
}

\begin{abstract}
The research of the integration of kindergarten curriculum and game has become the basic trend of infant education reform. Kindergarten curriculum is a practical carrier that carries the infant education goal and education value and the game is an important form for the all-around development education of infants. The effective integration of kindergarten curriculum and game should be realized from pursuit of common value goal, generation of "self-organization" function and creation of suitable environment to promote the all-around and harmonious development of infants.

Curriculum integration is an important educational development trend derived from the contemporary integrated science and technology trend. Essentially, "the goal of education is to shape people in a wide sense or accurately help children become perfect people”. This means the learning and development of infants is integral and based on the all-around physical, emotional, perceptive and social development. The kindergarten curriculum, as a practical carrier that carries the education goal and education value, should built an open, integral and changing curriculum integration system for the purpose of adapting to the needs of times and infant development. According to the spirit of Guidance for Kindergarten Education, "game is an important form of all-around development education of infants"; "with the game as the basic activity, the education should be integrated into the various activities." Based on this, it is necessary to complete interpret the existence of game and its value for kindergarten curriculum and strive to realize the effective integration of kindergarten curriculum and game.
\end{abstract}

\section{Relationship between kindergarten curriculum and game}

What should the relationship between kindergarten curriculum and game be? At present, different people have different views on the problem with regard to kindergarten curriculum, so there is different understanding of the relationship between kindergarten curriculum and game under different curriculum outlook as follows:

(I) Kindergarten curriculum is the learning subjects

This definition highlights that the task of kindergarten education is to pass on the selected systematic knowledge to infants, in which the "teaching" characterized by branched curriculum is the main form of kindergarten education and the game is often the medium and carrier of passing on, consolidating and enhancing the knowledge. The kindergarten curriculum and game are separated, without crossing relation in terms of content.

(II) Kindergarten curriculum is the activity

It is thought that "kindergarten curriculum is the sum of kindergarten education activities" from the perspective of curriculum design and implementation. The game, as the "basic activity" of kindergarten, naturally becomes an important part or content of kindergarten curriculum and a structural factor of kindergarten curriculum, rather than a "form".

(III) Kindergarten curriculum is the learning experience

This definition from the perspective of infants focuses on what and how the infants gain from the kindergarten as well as the infant needs, interest and subjectivity cultivation. In this opinion, the game is classified into the content of kindergarten curriculum and also a realistic practical form of kindergarten teaching. 


\section{Necessity of the effective integration of kindergarten curriculum and game}

(I) Integration is the need of kindergarten curriculum development

Since the founding of new China, affected by Soviet education, China's kindergarten curriculum focuses on teaching infants subject knowledge systematically by steps according to the infant's age and feature distribution, that is, subject curriculum. This curriculum has strong internal logic structure for the convenience of infant's systematic and progressive learning. However, while attaching great importance to the subject systematicness, the subject curriculum has a series of problems, such as rational curriculum objective, separated curriculum content, neglect of the integrity of infant life, result-oriented curriculum evaluation and neglect of the development of infant in perception, emotion, personality and other aspects. To change this situation, a series of documents and laws with regard to infant education are formulated, in which Guidance for Kindergarten Education (Trial) points out that the education content of kindergarten is comprehensive and enlightening and can be divided into health, language, society, science and arts. The document highlights that the content of each field is interpenetrative to promote the development of infant in emotion, attitude, ability, knowledge, skills and other aspects from different perspective. Guide to Learning and Development of 3-6 Year Old Children also emphasizes that attention should be paid to the interpenetration and integration between fields and objectives upon implementation to promote the all-around harmonious development of infants. Therefore, the kindergarten curriculum integration is imperative.

(II) Necessary relationship between game and kindergarten curriculum conforms to the integration needs

"The history of the application of game in the children education field shows that the game is not o pure natural activity any more once it enters the children education field, which will become an education activity due to the education values and education influences. To a certain degree, the systematic and normalized game activity is the curriculum content of kindergarten, rather than a "form" of curriculum implementation." Therefore, kindergarten curriculum occurs as a whole, but is penetrated by game. The natural integrity of game can generate and integrate the curriculum and the curriculum integration can be realized by game. There is necessary relationship between game and kindergarten curriculum.

\section{Concrete strategy of effective integration of kindergarten curriculum and game}

(I) Realize the effective integration of kindergarten curriculum and game in the pursuit of common value goal

Kindergarten education activity is not only a value creation activity, but also a value selection activity. The integration of kindergarten curriculum and game should pay attention to the value core hidden in the two different activities, that is, value goal selection problem. From the history of kindergarten curriculum development, we can see there is a shifting relationship between the position of game in the kindergarten curriculum and the emphasis on knowledge and skills. When the knowledge transmission is emphasized in the kindergarten curriculum, the game must be neglected. The main cause is rooted in the traditional knowledge-based curriculum outlook. The knowledge-based curriculum outlook regards the curriculum "as a set of subjective knowledge system selected by the adult for young people and waiting for the "occupation" or "mastering" of young people", with a metaphor of "preparation". The kindergarten curriculum is a preparation for primary school curriculum, leading to the teaching of knowledge and skills in advance. However, the game and curriculum are separated for a long time under this curriculum outlook and the game is often regarded a the relaxation and rest after learning.

With the social development and continuous improvement of kindergarten education practice, people realize that the current situation, trend and need of human and social development in today's world require that a complete education value must be kept to shape a complete education because only the complete education can promote the human and society to develop towards humanity 
harmoniously and independently, namely, the modern education value should develop towards the direction of promoting the sustainable development of human and the society. Therefore, the "people"-based curriculum outlook is gradually concerned, which pays attention to the development of infants in initiative, independence and creative, thus embodying the unique value of game as the "human activity". The game can provide a form with development suitability for the initiative learning and experience construction of infants and meet and develop the children in a natural form of life entity at most. The value commonality becomes the precondition of the effective integration of kindergarten curriculum and game and also provides more possibilities of realization and ideological preparation and direction for the integration of the two by teaching staff.

(II) Realize the effective integration of kindergarten curriculum and game in the generation of "self-organization" function

German theoretical physicist H.Haken divides the organization into self-organization and other-organization by the evolutionary form of organization, in which the latter one is that an organization is organized by relying on external command and the former one is that the structured system is formed from disorder to order automatically by self organization, creation and evolution without any external command. Self-organization is an existence form of system and conforms to the survival mechanism of system more compared with other-organization because it, which is a natural energy, embodies the autonomy, openness, order and uncontrollability of system, exists under the action of others parts and for other parts and the whole and can coordinate the parts of system; each part interacts and forms a whole due to their causal association, which can gradually create rich complex forms and structures. At the same time, it can integrate the individual factor and social culture factor and put the opinions into various combinations to realize collision and coordination during dialogue and exchange so as to make the system become a generating open system with self-organization ability.

According to the self-organization theory, the kindergarten curriculum and game can be integrated through the generation of "self-organization" function because it is not a real "integration" if the kindergarten curriculum and game forms time, spatial and functional integration passively. The external cause must work by internal curriculum. With the social progress and physical and mental development needs of infants, the kindergarten curriculum continuously accepts the influence of external information with an open mind and adjusts and revises the aspect not compliance with the value ideal or presents new construction to achieve the self-organization process of internal restructuring by the interaction between inside and outside. As the real natural learning activity, the game can provide the selection and innovation possibility and flexibility for the unique experience construction of infants in the process interacting with the ambient environment. The infants can explore, discover, plan, think and actively build their experience and significance in the game and also form learning community around the interested question in the game. At that time, the game enriches and supplements the curriculum, which can not only be the "self-organization" process of the learning or experience construction of infants, but also become the source of kindergarten curriculum generation and development. The kindergarten curriculum and game become an open channel for personal transformation, with effective integration.

(III) Realize the effective integration of kindergarten curriculum and game in the creation of suitable environment

Good environment is a beautiful living space with the teaching staff's intention and wisdom and children's interest, which constitutes a tactic \& dynamic, material and mental children activity scene. The environment panning and layout is not only the cornerstone of kindergarten curriculum, but also the necessary condition for game. Therefore, the effective integration of kindergarten and game should realize the organic integration of environment and create a supporting and inclusive environment that can provide operation, transformation and adjustment for infants and that they can bring them into their experience system. The environment mainly includes two aspects: provision of suitable conditions and creation of environment (manly game environment).

1. Site

Site is a miniature world for infants, where they can have various sense experiences. Therefore, 
the teacher should give play to their subjective initiative according to the actual situation to provide diversified sites for infants. It is required to not only design an open and interactive playground for infants, but also provide a hidden learning space for infants; there should be not only noisy dynamic corner (building block corner and drama corner), but also stable and fixed static corner (book corner and science corner). The open and hidden, dynamic and static, determined and undetermined layout can make children wander in the game world and the corners in series enhance their functions and also encourages the active interaction and growth of infants.

2. Time

Time is a powerful guarantee for the sufficient game of infants. To make infants do all kinds of high-level games, it is required to consider whether the time is reasonable and whether it can make infants give play to the game skills. The research shows that infants like the game more than 30 minutes and the long-term game can improve the children's perception. The teacher should provide suitable time and the time of creative game should reach a certain ratio. The guarantee of insufficient game time of infants is essential to the integration of kindergarten curriculum and game and the strict boundary between curriculum and game should be broken to make infants actively explore and learn in the curriculum teaching form and build their knowledge during the interaction with materials and toys. The game environment with education significance can be created combined with the curriculum goal and content to make the game become the curriculum teaching form and curriculum content.

3. Game toys and materials

Game toys and materials are not only the game partner of infants, but also their learning "tools" as well as the real embodiment of teacher's educational intention. Therefore, the teacher is required to get close to the infants' living experience and the theme of curriculum development and choose the toys and materials that the infants are interested in and willing to operate and explore using their hand and brain in choosing the game toys and materials. In addition, in the input and management, the teacher should provide corresponding game materials according to the age of infants and change the playing methods timely.

\section{Acknowledgments}

Source of project: "Research on Development of Kindergarten Game Curriculum Based on Traditional Games of Yi Children”, Humanities \& Social Science Base of Sichuan Provincial Education Department --Yi Culture Research Center Project

\section{References}

[1] Zhang Huanting. Selected Readings in Books on Schools of Educational Thought of Modern Western Bourgeois[M]. Beijing: People's Education Press, 1980:289

[2] Feng Xiaoxia. Thinking and Trying on Kindergarten curriculum Construction Based on Activity Theory[J]. Preschool Education Studies Journal Agency, 1997 (4).

[3] Yuan Guiren. New Encyclopedia of Chinese Teacher[M]. Encyclopedia of China Publishing House, 2003:471

[4] Li Jimei, Feng Xiaoxia. Guide to Learning and Development of 3-6 Year Old Children[M]. People's Education Press, 2013:39

[5][6] Liu Yan. Kindergarten Game Teaching Theory[M]. Beijing: China Social Science Press, 1999:97,280

[7] Liu Yan. General Theory of Children Game[M]. Beijing Normal University Publishing House, 2004:359

[8] Hu Zhongping, Liu Chaohui. Challenge and Response: Outlook on Educational Goal in the 20th Century[M]. Jinan: Shandong Education Press, 1995: 503 